\title{
Mathematisches zur Theorie der Linienbreite
}

\author{
Marianne Friedrich \\ Universität Zürich
}

Eingegangen am 26. Januar 1966

\begin{abstract}
In the present paper we consider the emission of light quanta by an atom using the methods of non-relativistic quantum theory. The differential equations occurring there are solved using a Laplace transformation. By means of an analytic continuation and a displacement of the integration path we get exact solutions whose approximations lead to the usual formulae for the intensity distribution and for the natural line width.
\end{abstract}

\section{§ 1. Einleitung}

Nach den Erkenntnissen der Elektrodynamik sendet ein angeregtes Atomelektron Licht aus. Die Lichtwellen sind gedämpft und nicht monochromatisch. Sie können aber als Superposition von periodischen Wellen aufgefaßt werden. Die Intensität der Partialwellen mit der Frequenz $v$ beträgt näherungsweise ([1], S. 33)

$$
I(v)=I_{0} \frac{\gamma / 2 \pi}{\left(v-v_{0}\right)^{2}+\gamma^{2} / 4} .
$$

Hierbei ist $v_{0}$ die ungestörte Schwingungsfrequenz und $\gamma$ die Dämpfungskonstante. Die Konstante $I_{0}$ ist so gewählt, daß sie gleich der gesamten Intensität $\int I(v) d v$ ist.

Die Lichtemission eines angeregten Atoms wurde zuerst von WeIssKOPF und Wigner ([2], S. 54) untersucht, welche die Formel (1.1) theoretisch begründet haben, wobei die auftretenden Differentialgleichungen durch einen Ansatz näherungsweise gelöst wurden. HeITLER und $\mathrm{MA}_{\mathrm{A}}$ [3] gaben eine exakte Lösung mit Hilfe einer Fourier-Transformation. Diese Methode wurde von Arnous und Heitler [4], [5] in eine allgemeine Theorie über die Linienbreite eingebaut. Dabei stellte es sich heraus, daß die Größe $\gamma$ nicht konstant, sondern eine Funktion der Frequenz ist.

Eine weitere wichtige Arbeit ist der Artikel von KäLLÉN [6] über die natürliche Linienbreite. Hier werden die Differentialgleichungen nicht durch einen Ansatz gelöst, sondern mit Hilfe der Laplacetransformation in eine einfachere Form übergeführt. In der vorliegenden Arbeit soll die Methode von KäLLÉn modifiziert werden. Durch analytische Fort23 Commun. math. Phys., Vol. 2 
setzung und Änderung des Integrationsweges erhalten wir eine exakte Formel für die Intensitätsverteilung von der Form

$$
I(v)=(1.1)+\text { kleine Korrekturen } .
$$

\section{§ 2. Die Wahrscheinlichkeitsamplituden}

Die Emission von Licht ist eine Folge der Wechselwirkung zwischen Elektron und elektromagnetischem Feld. Dieses physikalische System kann verschiedene Zustände annehmen, die in unserem Fall die folgenden sein sollen:

Zur Zeit $t=0$ wird der Zustand $\Psi_{0}$ realisiert, in dem es ein Elektron mit der Energie $E_{0}$, aber noch kein Lichtquant gibt. Dieser Zustand heiße Vakuumzustand. Zu späteren Zeiten ist noch ein zweiter Zustand möglich. Das Elektron hat keine Energie mehr, es existiert aber ein Photon mit der Frequenz $v$ und der Energie $\hbar v$, die aber nicht gleich $E_{0}$ sein muß. Dieser Einteilchenzustand wird mit $\Psi_{1}$ bezeichnet.

Unser Ziel ist es, die Wahrscheinlichkeit zu berechnen, mit der sich das System zu einem beliebigen Zeitpunkt im Vakuum- oder in einem Einteilchenzustand befindet. Dazu bestimmen wir zuerst die Wahrscheinkeitsamplituden $a_{0}(t)$ und $a_{\lambda}(t)$, deren Normquadrate die Wahrscheinlichkeit angeben, daß sich das System zur Zeit $t$ im Vakuum- oder im Einteilchenzustand mit einem Photon der Frequenz $v_{\lambda}$ befindet.

Wenn wir annehmen, daß die Energiewerte des Lichtquants diskret sind, so daß es ein diskretes Frequenzspektrum $v_{1}, v_{2}, \ldots$ gibt, und wenn wir $E_{0} / \hbar=v_{0}$ und $\hbar=c=1$ setzen, können die Wahrscheinlichkeitsamplituden berechnet werden:

$$
\begin{aligned}
& a_{0}(t)=\frac{1}{2 \pi} \int_{\gamma-i \infty}^{\gamma+i \infty} \frac{e^{p t}}{i p-v_{0}-\sum_{\varkappa \neq 0} \frac{\left|H_{\chi}\right|^{2}}{i p-v_{\varkappa}}} d p \\
& a_{\lambda}(t)=\frac{1}{2 \pi} \int_{\gamma-i \infty}^{\gamma+i \infty} \frac{H_{\lambda}^{*} \cdot e^{p t}}{\left(i p-v_{\lambda}\right)\left[i p-v_{0}-\sum_{\varkappa \neq 0} \frac{\left|H_{\varkappa}\right|^{2}}{i p-v_{\varkappa}}\right]} d p .
\end{aligned}
$$

Diese Formeln lassen sich mit Hilfe einer Laplacetransformation [7], die für $\gamma=0$ in eine Fouriertransformation übergeht, aus den Gleichungen ([1], S. 137)

$$
i \hbar \dot{b}_{n}(t)=\sum_{m} H_{\operatorname{int} n / m}^{\prime} b_{m}(t)
$$

herleiten, wobei $H_{\operatorname{int} n / m}^{\prime}$ das Matrixelement des Wechselwirkungsoperators für einen Übergang zwischen zwei möglichen Zuständen ist. Diese Matrixelemente heißen bei uns $H_{\lambda}$. 
Die Formeln (2.1), die auch aus KäLLÉNs Beziehungen (28.24) und (28.18) ([6], S. 276, 277) folgen, verallgemeinern wir nun für das kontinuierliche Frequenzspektrum. Dabei geht die Summe $\sum \frac{\left|H_{x}\right|^{2}}{i p-v_{\varkappa}}$ über in das Integral $\int \frac{|H(v, \Omega)|^{2} \varrho(v)}{i p-\nu} d v d \Omega$, in $\operatorname{dem} \varrho(v) d v d \Omega$ die Zahl der Wellen angibt, deren Frequenzen zwischen $v$ und $v+d v$, und deren Fortpflanzungsrichtungen im Raumwinkel $d \Omega$ liegen. Nun setzen wir

$$
\int_{0}^{4 \pi}|H(v, \Omega)|^{2} \varrho(v) d \Omega=f(v) .
$$

Falls $\frac{1}{v}$ groß ist gegenüber dem Radius des Atoms, ist $f(v)$ proportional $\mathrm{zu} v$, wobei die Proportionalitätskonstante sehr klein ist ([1], S. 42 und [2], S. 66). Für große $v$, die uns nicht mehr interessieren, da sie ja nicht mehr im sichtbaren Bereich liegen, setzen wir willkürlich $f(v)=C \cdot \nu^{-\alpha}$ mit $\alpha>1$. Somit ist

$$
f(v)=\begin{array}{cl}
C_{1} \cdot v \text { für } v \leqq N & \text { mit } C=C_{1} N^{\alpha+1} \\
C \cdot v^{-\alpha} \text { für } v \geqq N & \text { und } \alpha>1 .
\end{array}
$$

Die Funktion $f(v)$ ist also reell mit positiven Werten, langsam veränderlich und für alle $v \neq N$ differenzierbar ([2], S. 63). Sie ist für $v<N$ eine analytische Funktion, die sich ohne weiteres in die komplexe Ebene fortsetzen läßt.

$$
f(p)=C_{1} \cdot p \quad \text { falls } \quad R e(p) \leqq N .
$$

Um die Ausdrücke (2.1) auf das kontinuierliche Spektrum umzuschreiben, haben wir also $\sum \frac{\left|H_{x}\right|^{2}}{i p-v_{\varkappa}}$ durch

$$
\Gamma(p)=\int_{0}^{\infty} \frac{f(\nu)}{i p-v} d \nu
$$

zu ersetzen, so daß wir folgende Darstellung der Wahrscheinlichkeitsamplituden erhalten:

$$
\begin{aligned}
a_{0}(t) & =\frac{1}{2 \pi} \lim _{M \rightarrow \infty} \int_{\gamma-i M}^{\gamma+i M} \frac{e^{p t}}{i p-\nu_{0}-\Gamma(p)} d p \\
a(\nu, t) & =\frac{1}{2 \pi} \lim _{M \rightarrow \infty} \int_{\gamma-i M}^{\gamma+i M} \frac{H^{*}(\nu) \cdot e^{p t}}{(i p-\nu)\left[i p-v_{0}-\Gamma(p)\right]} d p .
\end{aligned}
$$

\section{§ 3. Die Funktion $\boldsymbol{\Gamma}(\boldsymbol{p})$}

Wir haben im vorangehenden Kapitel formal das Integral

$$
\Gamma(p)=\int_{0}^{\infty} \frac{f(v)}{i p-v} d \nu
$$


gebildet, ohne uns darum zu kümmern, ob dieses uneigentliche Integral existiere, und ob $\Gamma(p)$ analytisch sei. Diese Fragen werden wir zunächst abklären.

Das uneigentliche Integral existiert, falls der Integrand für $0 \leqq v<\infty$ stetig ist, und falls es eine Zahl $M$ gibt, so daß

$$
J_{1}=\left|\int_{\mu_{1}}^{\mu_{2}} \frac{f(v)}{i p-v} d v\right| \leqq \varepsilon \quad\left(0 \leqq \mu_{1}<\mu_{2}\right),
$$

sobald $\mu_{1}$ und $\mu_{2}$ größer als eine von $\varepsilon$ abhängige Zahl $M$ sind.

Da $f(v)$ eine stetige Funktion ist, wird die erste Bedingung von allen $p$ erfüllt, für die $i p-v \neq 0$, d. h. $p \neq-i v$. Der Integrand ist also für alle komplexen Zahlen $p$, deren Bildpunkte nicht auf dem negativen Teil der imaginären Achse liegen, stetig. Diese Zahlen bilden die Menge $\bar{E}$.

Zur Bestimmung der Konstanten $M$ schätzen wir $J_{1}$ für ein beliebiges, aber festes $p=a+i b$ ab.

$$
J_{1} \leqq \int_{\mu_{1}}^{\mu_{2}} \frac{f(v)}{|i p-v|} d \nu .
$$

Es ist $|i p-v|^{2}=(b+v)^{2}+a^{2}$. Für große $v$ verhält sich $f(v)$ nach (2.2) wie $C \cdot v^{-\alpha}$ mit $\alpha>1$. Somit ist

$$
J_{1} \leqq \int_{\mu_{1}}^{\mu_{2}} \frac{C \cdot v^{-\alpha}}{\sqrt{(b+v)^{2}+a^{2}}} d v \text { für } \mu_{1} \geqq N .
$$

Für die weitere Abschätzung lassen wir nur solche $p$ zu, deren Imaginärteile $b$ positiv sind. Dann ist $b+v>0$ und somit $\sqrt{(b+v)^{2}+a^{2}}>b+$ $+v>v$. Nun läßt sich (3.1) weiter abschätzen.

Also ist

$$
J_{1} \leqq \int_{\mu_{1}}^{\mu_{2}} \frac{C \cdot v^{-\alpha}}{\nu} d \nu=C \cdot \frac{1}{\alpha}\left[\frac{1}{\mu_{1}^{\alpha}}-\frac{1}{\mu_{2}^{\alpha}}\right]<C \cdot \frac{1}{\mu_{1}^{\alpha}} .
$$

$$
J_{1} \leqq \frac{C}{\mu_{1}^{\alpha}} \leqq \varepsilon \quad \text { falls }\left(\frac{1}{\mu_{1}}\right)^{\alpha} \leqq \frac{\varepsilon}{C}, \quad \text { d. h. } \quad \mu_{1} \geqq\left(\frac{C}{\varepsilon}\right)^{\frac{1}{\alpha}}=M^{\prime}(\varepsilon) .
$$

Das Integral $J_{1}$ konvergiert also gegen 0 , sobald $\mu_{1}, \mu_{2} \geqq M(\varepsilon)=$ $=\max \left(N, M^{\prime}(\varepsilon)\right)$. Da die Konstanten $N$ und $M^{\prime}$ von der Wahl der komplexen Zahl $p$ unabhängig sind, existiert die Funktion $\Gamma(p)$ für alle $p$, deren Bildpunkte in der oberen Hälfte der komplexen Ebene liegen, gleichmäßig.

Wenn hingegen der Imaginärteil von $p$ negativ oder Null ist, so gilt $(b+v)^{2}>v^{2}$ nur für $v<-\frac{b}{2}$. Für $b \leqq 0$ müssen wir den Ausdruck (3.1) also etwas genauer abschätzen. 
Wir wählen $\mu_{1}$ so groß, daß $\mu_{1}>-b$. Nun ist $b+v>0$, und wir können die Abschätzung von (3.1) weiterführen.

$$
J_{1} \leqq \int_{\mu_{1}}^{\mu_{2}} \frac{C \cdot \nu^{-\alpha}}{b+v} d \nu \leqq \int_{\mu_{1}}^{\mu_{2}} \frac{C(b+v)^{-\alpha}}{b+\nu} d \nu=\frac{C}{\alpha}\left[\left(\frac{1}{b+\mu_{1}}\right)^{\alpha}-\left(\frac{1}{b+\mu_{2}}\right)^{\alpha}\right]
$$

Somit ist

$$
\begin{gathered}
J_{1} \leqq C\left(\frac{1}{b+\mu_{1}}\right)^{\alpha} \leqq \varepsilon \text { sobald } b+\mu_{1} \geqq\left(\frac{C}{\varepsilon}\right)^{\frac{1}{\alpha}} \text {,d.h. } \mu_{1} \geqq\left(\frac{C}{\varepsilon}\right)^{\frac{1}{\alpha}}-b=M^{\prime \prime}(\varepsilon) \\
J_{1} \leqq \varepsilon \text { sobald } \mu_{1} \geqq M(\varepsilon)=\max \left(N, M^{\prime \prime}(\varepsilon)\right) .
\end{gathered}
$$

Die Funktion $\Gamma(p)$ ist also auch für diejenigen $p \in \bar{E}$ definiert, deren Imaginärteile nicht positiv sind. Zwar ist die Konvergenz nicht gleichmäßig $\left(M^{\prime \prime}(\varepsilon)\right.$ hängt auch von $\left.b a b\right)$, aber wir werden sehen, daß durch eine zusätzliche Bedingung gleichmäßige Konvergenz erreicht werden kann.

Wir betrachten nun solche $p$, deren Imaginärteile größer als eine feste negative Zahl $b_{1}$ sind. Nun wiederholen wir die vorangehende Abschätzung, wobei wir $\mu_{1}>-b_{1}$ wählen. Dann ist

$$
\begin{gathered}
b+v>b_{1}+v>b_{1}+\mu_{1}>0 \\
(b+v)^{2}>\left(b_{1}+v\right)^{2} .
\end{gathered}
$$

Nun konvergiert $J_{1}$ gegen 0 , sobald $\mu_{1}$ größer als $N$ und $M^{\prime \prime}(\varepsilon)=\left(\frac{C}{\varepsilon}\right)^{\frac{1}{\alpha}}-$ - $b_{1}$. Im Gegensatz zu vorher ist $M^{\prime \prime}(\varepsilon)$ nun von $b$ unabhängig. Das Integral $\Gamma(p)$ existiert also für alle $p$ mit $\operatorname{Im}(p)>b_{1}$ gleichmäßig.

Zusammengefaßt besitzt $\Gamma(p)$ folgende Eigenschaften: Die Funktion $\Gamma(p)$ existiert gleichmäßig für alle $p$, deren Bildpunkte oberhalb einer Parallelen zur reellen Achse, aber nicht auf der negativen imaginären Achse liegen. Insbesondere haben wir auch gleichmäßige Existenz, falls die Bildpunkte in einem beliebigen Rechteck liegen, dessen Seiten parallel zu den Achsen sind, und das die negative imaginäre Achse nicht schneidet, das also ganz in $\bar{E}$ liegt.

Nun gilt folgender Satz ([8], S. 317, 321): Wenn der Grenzwert $\lim _{a \rightarrow \infty} g(a, p)$ in jedem Rechteck der Ebene $\bar{E}$ gleichmäßig in $p$ existiert, und wenn $g(\alpha, p)$ eine analytische Funktion von $p$ für alle $p \in \bar{E}$ ist, so ist auch $\lim _{a \rightarrow \infty} g(a, p)$ eine analytische Funktion von $p$. Wir setzen nun

$$
g(a, p)=\int_{0}^{a} \frac{f(v)}{i p-v} d v .
$$

Da $f(v)$ stetig ist, so ist $g(a, p)$ nach dem Hauptsatz der Funktionentheorie ([8], S. 296) eine analytische Funktion für alle $p$ mit $i p \neq v, \mathrm{~d}$. h. 
für alle $p \in \bar{E}$. Ebenso existiert der Grenzwert des Integrals $\int_{0}^{a} \frac{f(v)}{i p-v} d v$ gleichmäßig in jedem Rechteck, das ganz in $\bar{E}$ liegt. Daher ist $\Gamma(p)$ für alle $p \in \bar{E}$ eine analytische Funktion.

\section{§ 4. Die analytische Fortsetzung von $\boldsymbol{\Gamma}(\boldsymbol{p})$}

Nach den vorangehenden Überlegungen ist es klar, daß

$$
\Gamma(p)=\int_{0}^{\infty} \frac{f(v)}{i p-v} d v
$$

nur dann sinnvoll ist, wenn $i p$ reell und negativ oder nicht reell ist. Falls hingegen $i p$ reell und nicht negativ ist, so ist der Integrand nicht auf dem ganzen Integrationsweg beschränkt, so daß das Integral singulär wird. Für diese Werte $p_{0}$ können wir aber Funktionen definieren, die regulär sind, indem wir den Integrationsweg geeignet wählen (Fig. 1).

$\Gamma_{+}\left(p_{0}\right)=\int_{C_{+}} \frac{f(v)}{i p_{0}-v} d v \quad p$ nähert sich von rechts der imaginären Achse, $\Gamma_{-}\left(p_{0}\right)=\int_{C_{-}} \frac{f(v)}{i p_{0}-v} d v \quad p$ nähert sich von links der imaginären Achse.

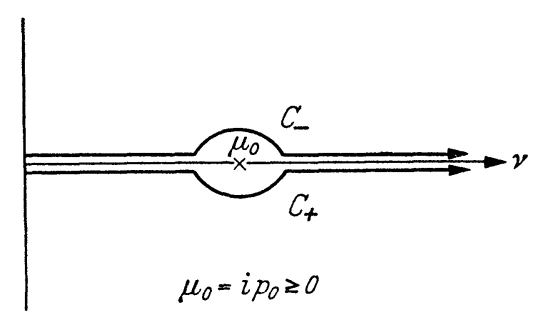

Fig. 1

Um den Zusammenhang zwischen $\Gamma_{+}\left(p_{0}\right)$ und $\Gamma_{-}\left(p_{0}\right)$ zu finden, betrachten wir die Differenz

$$
\Delta_{\varrho} \Gamma\left(p_{0}\right)=\Gamma\left(p_{0}-\varrho\right)-\Gamma\left(p_{0}+\varrho\right)
$$

in der wir $\varrho$ gegen 0 konvergieren lassen. Da die Funktion $\Gamma(p)$ für alle $p$ mit positivem Imaginärteil analytisch ist, untersuchen wir diesen Grenzwert nur für solche $p_{0}$, deren Bildpunkte auf der negativen imaginären Achse liegen. Für die folgenden Überlegungen ist daher $i p_{0}$ $=\mu_{0} \geqq 0$. 
Auf Grund der Beziehung (2.4) erhalten wir:

$$
\begin{aligned}
\Delta_{\varrho} \Gamma\left(p_{0}\right) & =\int_{0}^{\infty} f(\nu)\left[\frac{1}{i\left(p_{0}-\varrho\right)-v}-\frac{1}{i\left(p_{0}+\varrho\right)-v}\right] d v \\
& =\int_{0}^{\infty} f(v) \frac{2 i \varrho}{\left(i p_{0}-v\right)^{2}-(i \varrho)^{2}} d \nu \\
\Delta_{\varrho} \Gamma\left(p_{0}\right) & =2 i \int_{0}^{\infty} f(v) \frac{\varrho}{\left(\mu_{0}-v\right)^{2}+\varrho^{2}} d \nu .
\end{aligned}
$$

Bevor wir $\varrho$ gegen 0 gehen lassen, werden wir die gleichmäßige Existenz des Integrals (4.2) für $\mu_{0} \geqq 0$ nachweisen. Dazu muß der Integrand stetig sein. Diese Bedingung ist sicher erfüllt, da $f(v)$ stetig und $\left(\mu_{0}-\right.$ $-v)^{2}+\varrho^{2}>0$ ist. Außerdem haben wir zu zeigen, daß es eine von $\varrho$ unabhängige Zahl $M$ gibt, so daß

$$
J_{2}=\left|\int_{\mu_{1}}^{\mu_{2}} \frac{f(\nu) \varrho}{\left(\mu_{0}-v\right)^{2}+\varrho^{2}} d v\right| \leqq \varepsilon
$$

ist, sobald $\mu_{1}, \mu_{2}$ größer als $M(\varepsilon)$ sind.

Es sei $\mu_{2}>\mu_{1} \geqq N$, so daß sich $f(v)$ wie $C v^{-\alpha}$ mit $\alpha>1$ verhält. Außerdem soll $\mu_{1}>\mu_{0}$ sein, so daß $\nu-\mu_{0} \geqq \mu_{1}-\mu_{0}>0$. Auch schränken wir $\varrho$ auf das abgeschlossene Intervall $I=\langle 0,1\rangle$ ein. Somit ist

$$
J_{2} \leqq \int_{\mu_{1}}^{\mu_{2}} \frac{f(v) \cdot \varrho}{\left(\mu_{0}-v\right)^{2}+\varrho^{2}} d v \leqq \int_{\mu_{1}}^{\mu_{2}} \frac{C \cdot v^{-\alpha}}{\left(\mu_{0}-v\right)^{2}} d v
$$

Da $\alpha$ und $\mu_{0}$ positiv sind, gilt $\left(\nu-\mu_{0}\right)^{-\alpha} \geqq \nu^{-\alpha}$. Somit ist

$$
\begin{aligned}
C \cdot \int_{\mu_{1}}^{\mu_{2}} & \frac{v^{-\alpha}}{\left(\mu_{0}-v\right)^{2}} d v \leqq C \int_{\mu_{1}}^{\mu_{2}} \frac{\left(\nu-\mu_{0}\right)^{-\alpha}}{\left(\nu-\mu_{0}\right)^{2}} d \nu \\
& =C \cdot \frac{1}{\alpha+1} \cdot\left[\left(\frac{1}{\mu_{1}-\mu_{0}}\right)^{\alpha+1}-\left(\frac{1}{\mu_{2}-\mu_{0}}\right)^{\alpha+1}\right]<C \cdot\left(\frac{1}{\mu_{1}-\mu_{0}}\right)^{\alpha+1} .
\end{aligned}
$$

Also ist

$$
\begin{gathered}
J_{2} \leqq C\left(\frac{1}{\mu_{1}-\mu_{0}}\right)^{\alpha+1} \leqq \varepsilon \text { sobald } \frac{1}{\mu_{1}-\mu_{0}} \leqq\left(\frac{\varepsilon}{C}\right)^{\frac{1}{\alpha+1}} \\
\text { d. h. } \mu_{1} \geqq \mu_{0}+\left(\frac{C}{\varepsilon}\right)^{\frac{1}{\alpha+1}}=M^{\prime}(\varepsilon) .
\end{gathered}
$$

Es gibt daher eine von $\varrho$ unabhängige Konstante $M(\varepsilon)$, so daß

$$
J_{2}=\left|\int_{\mu_{1}}^{\mu_{2}} \frac{f(v)}{\left(\mu_{0}-v\right)^{2}+\varrho^{2}} d \nu\right| \leqq \varepsilon \quad \text { für } \quad \mu_{2}>\mu_{1} \geqq M(\varepsilon)=\max \left(N, M^{\prime}(\varepsilon)\right) .
$$


Nachdem wir nun bewiesen haben, daß für $0<\varrho \leqq 1$ die Differenz $\Delta_{\varrho} \Gamma\left(p_{0}\right)$ gleichmäßig in $\varrho$ existiert, lassen wir $\varrho$ gegen 0 konvergieren.

$$
\Delta \Gamma\left(p_{0}\right)=\lim _{\varrho \rightarrow 0} \Delta_{\varrho} \Gamma\left(p_{0}\right)=2 i \cdot \lim _{\varrho \rightarrow 0} \int_{0}^{\infty} f(v) \frac{\varrho}{\left(\mu_{0}-v\right)^{2}+\varrho^{2}} d v .
$$

Zur Berechnung der Sprungfunktion $\Delta \Gamma\left(p_{0}\right)$ zerlegen wir das Integral (4.3).

$$
\begin{aligned}
& \lim _{\varrho \rightarrow 0} \int_{0}^{\infty} f(v) \frac{\varrho}{\left(\mu_{0}-v\right)^{2}+\varrho^{2}} d v=\lim _{\varrho \rightarrow 0} \int_{0}^{\mu_{0}-\tau} \frac{f(v) \cdot \varrho}{\left(\mu_{0}-v\right)^{2}+\varrho^{2}} d v, \\
& +\lim _{\varrho \rightarrow 0} \int_{\mu_{0}-\tau}^{\mu_{0}+\tau} \frac{f(v) \cdot \varrho}{\left(\mu_{0}-v\right)^{2}+\varrho^{2}} d v+\lim _{\varrho \rightarrow 0} \int_{\mu_{0}+\tau}^{\infty} \frac{f(v) \cdot \varrho}{\left(\mu_{0}-v\right)^{2}+\varrho^{2}} d v .
\end{aligned}
$$

$\tau$ sei vorläufig eine positive reelle Zahl. Auch beschränken wir uns auf Werte $p_{0}$ mit negativem Imaginärteil $\left(\mu_{0}>0\right)$. Zuerst berechnen wir den Grenzwert

$$
\lim _{\varrho \rightarrow 0} \int_{0}^{\mu_{0}-\tau} \frac{f(v) \cdot \varrho}{\left(\mu_{0}-v\right)^{2}+\varrho^{2}} d v .
$$

Der Integrand ist für $0 \leqq \nu \leqq \mu_{0}-\tau$ eine stetige Funktion von $v$. Da die Funktion $f(v)$ im abgeschlossenen Intervall $\left\langle 0, \mu_{0}\right\rangle$ stetig ist, gibt es eine nur von $\mu_{0}$ abhängende Konstante $F$, so daß $f(v) \leqq F$ ist auf diesem Intervall. Diese Abschätzung gilt natürlich auch für die $v$ aus dem Integrationsintervall, denn dieses ist im Intervall $\left\langle 0, \mu_{0}\right\rangle$ enthalten. Somit ist

$$
\left|f(v) \frac{\varrho}{\left(\mu_{0}-v\right)^{2}+\varrho^{2}}\right| \leqq \frac{F \cdot \varrho}{\left(\mu_{0}-v\right)^{2}} \leqq \frac{F \cdot \varrho}{\tau^{2}} \leqq \varepsilon \text { sobald } \varrho \leqq \frac{\varepsilon \cdot \tau^{2}}{F} .
$$

Also konvergiert der Integrand des ersten Gliedes von (4.4) für $\varrho \rightarrow 0$ gleichmäßig für alle $v$ gegen 0 . Es sind somit alle Voraussetzungen für die Vertauschung der Grenzwertbildung mit der Integration erfüllt ([9], Bd. II, S. 140).

$$
\lim _{\varrho \rightarrow 0} \int_{0}^{\mu_{0}-\tau} f(v) \frac{\varrho}{\left(\mu_{0}-v\right)^{2}+\varrho^{2}} d \nu=\int_{0}^{\mu_{0}-\tau} \lim _{\varrho \rightarrow 0} f(v) \frac{\varrho}{\left(\mu_{0}-v\right)^{2}+\varrho^{2}} d \nu=0 .
$$

Das erste Glied der Zerlegung (4.4) liefert also keinen Beitrag:

$$
\lim _{\varrho \rightarrow 0} \int_{0}^{\mu_{0}-\tau} \frac{f(v) \cdot \varrho}{\left(\mu_{0}-v\right)^{2}+\varrho^{2}} d v=0
$$

Nun haben wir das Glied

$$
\lim _{\varrho \rightarrow 0} \int_{\mu_{0}-\tau}^{\mu_{0}+\tau} f(\nu) \frac{\varrho}{\left(\mu_{0}-v\right)^{2}+\varrho^{2}} d \nu
$$

zu berechnen. Wir wissen, daß $f(v)$ für $\nu=\mu_{0}$ stetig ist.

$$
f(v)=f\left(\mu_{0}\right)+r(v) \quad \text { mit }|r(v)| \leqq \varepsilon \text { für }\left|v-\mu_{0}\right| \leqq \delta(\varepsilon) .
$$


Daher ist

$$
\begin{aligned}
\lim _{\varrho \rightarrow 0} \int_{\mu_{0}-\tau}^{\mu_{0}+\tau} f(\nu) \frac{\varrho}{\left(\mu_{0}-v\right)^{2}+\varrho^{2}} d \nu & =f\left(\mu_{0}\right) \lim _{\varrho \rightarrow 0} \int_{\mu_{0}-\tau}^{\mu_{0}+\tau} \frac{\varrho}{\left(\mu_{0}-v\right)^{2}+\varrho^{2}} d \nu+ \\
& +\lim _{\varrho \rightarrow 0} \int_{\mu_{0}-\tau}^{\mu_{0}+\tau} \frac{r(\nu) \cdot \varrho}{\left(\mu_{0}-v\right)^{2}+\varrho^{2}} d v .
\end{aligned}
$$

Für ein festes $\tau$ läßt sich der erste Summand berechnen:

$$
\begin{gathered}
\lim _{\varrho \rightarrow 0} \int_{\mu_{0}-\tau}^{\mu_{0}+\tau} \frac{\varrho}{\left(\mu_{0}-v\right)^{2}+\varrho^{2}} d \nu=\left.\lim _{\varrho \rightarrow 0} \operatorname{arctg} \frac{\nu-\mu_{0}}{\varrho}\right|_{\mu_{0}-\tau} ^{\mu_{0}+\tau} \\
=2 \cdot \lim _{\varrho \rightarrow 0} \operatorname{arctg} \frac{\tau}{\varrho}=2 \cdot \frac{\pi}{2}=\pi .
\end{gathered}
$$

Also ist

Das Restglied

$$
f\left(\mu_{0}\right) \lim _{\varrho \rightarrow 0} \int_{\mu_{0}-\tau}^{\mu_{0}+\tau} \frac{\varrho}{\left(\mu_{0}-v\right)^{2}+\varrho^{2}} d \nu=\pi \cdot f\left(\mu_{0}\right) .
$$

$$
R(\tau)=\lim _{\varrho \rightarrow 0} \int_{\mu_{0}-\tau}^{\mu_{0}+\tau} r(\nu) \frac{\varrho}{\left(\mu_{0}-\nu\right)^{2}+\varrho^{2}} d \nu
$$

wird direkt abgeschätzt. Da $f(v)$ nicht nur für $v=\mu_{0}$, sondern für alle $\nu$ aus dem abgeschlossenen Intervall $\left\langle\mu_{0}-\tau, \mu_{0}+\tau\right\rangle$ stetig ist, so muß auch $r(v)$ auf diesem Intervall stetig sein. Daher gibt es ein $\nu=\nu_{\tau}$, so daß $|r(v)| \leqq\left|r\left(\nu_{\tau}\right)\right|$ für alle $\nu \in\left\langle\mu_{0}-\tau, \mu_{0}+\tau\right\rangle$. Wir schränken nun $\tau$ so ein, daß $\tau \leqq \delta(\varepsilon)$ und somit $|r(\nu)| \leqq \varepsilon$ ist. Diese Abschätzung gilt insbesondere auch für $v_{\tau}$. Daher ist $\left|r\left(\nu_{\tau}\right)\right| \leqq \varepsilon$, sobald $\tau \leqq \delta(\varepsilon)$. Nun schätzen wir das Restglied ab. Zunächst ist

$$
\begin{gathered}
\left|\int_{\mu_{0}-\tau}^{\mu_{0}+\tau} r(\nu) \frac{\varrho}{\left(\mu_{0}-v\right)^{2}+\varrho^{2}} d \nu\right| \leqq \int_{\mu_{0}-\tau}^{\mu_{0}+\tau}|r(\nu)| \frac{\varrho}{\left(\mu_{0}-\nu\right)^{2}+\varrho^{2}} d \nu \leqq \\
\leqq\left|r\left(\nu_{\tau}\right)\right| \cdot \int_{\mu_{0}-\tau}^{\mu_{0}+\tau} \frac{\varrho}{\left(\mu_{0}-\nu\right)^{2}+\varrho^{2}} d \nu=2\left|r\left(\nu_{\tau}\right)\right| \operatorname{arctg} \frac{\tau}{\varrho} \leqq\left|r\left(\nu_{\tau}\right)\right| \cdot \pi .
\end{gathered}
$$

Diese Abschätzung ist unabhängig von $\varrho$, sie bleibt auch gültig, wenn $\varrho$ gegen 0 konvergiert. Daher genügt das Restglied der Ungleichung

$$
|R(\tau)| \leqq \pi \cdot \varepsilon \text { sobald } \tau \leqq \delta(\varepsilon) .
$$

Unter Verwendung von (4.6) bekommen wir

$$
\lim _{\varrho \rightarrow 0} \int_{\mu_{0}-\tau}^{\mu_{0}+\tau} f(\nu) \frac{\varrho}{\left(\mu_{0}-v\right)^{2}+\varrho^{2}} d \nu=\pi \cdot f\left(\mu_{0}\right)+R(\tau) .
$$


Schließlich haben wir noch das dritte Glied von (4.4) zu bestimmen.

$$
\lim _{\varrho \rightarrow 0} \int_{\mu_{0}+\tau}^{\infty} f(v) \frac{\varrho}{\left(\mu_{0}-v\right)^{2}+\varrho^{2}} d v=\lim _{\varrho \rightarrow 0} \lim _{b \rightarrow \infty} \int_{\mu_{0}+\tau}^{b} f(\nu) \frac{\varrho}{\left(\mu_{0}-v\right)^{2}+\varrho^{2}} d v .
$$

Es gilt nun der Satz ([9], Bd. II, S. 146):

$$
\lim _{\varrho \rightarrow 0} \lim _{b \rightarrow \infty} \int_{a}^{b} g(v, \varrho) d v=\lim _{b \rightarrow \infty} \lim _{\varrho \rightarrow 0} \int_{a}^{b} g(v, \varrho) d v
$$

falls $\lim _{b \rightarrow \infty} \int_{a}^{b} g(v, \varrho) d \nu$ gleichmäßig und $\lim _{\varrho \rightarrow 0} \int_{a}^{b} g(v, \varrho) d v$ gewöhnlich existieren.

Der Grenzwert $\lim _{b \rightarrow \infty} \int_{\mu_{0}+\tau}^{b} f(v) \frac{\varrho}{\left(\mu_{0}-v\right)^{2}+\varrho^{2}} d v$ existiert gleichmäßig für $0<\varrho \leqq 1$, da für dieselben Werte von $\varrho$ das Integral (4.2) gleichmäßig existiert. Nun berechnen wir noch den Grenzwert von $\int_{\mu_{0}+\tau}^{b} \frac{f(v) \cdot \varrho}{\left(\mu_{0}-v\right)^{2}+\varrho^{2}} d v$ für $\varrho \rightarrow 0$.

$$
\left|\int_{\mu_{0}+\tau}^{b} f(v) \frac{\varrho}{\left(\mu_{0}-v\right)^{2}+\varrho^{2}} d \nu\right| \leqq \int_{\mu_{0}+\tau}^{b} f(v) \frac{\varrho}{\left(\mu_{0}-v\right)^{2}+\varrho^{2}} d \nu .
$$

Da $f(v)$ im Integrationsintervall stetig ist, gibt es eine Konstante $F$, so daß $f(v) \leqq F$.

$$
\int_{\mu_{0}+\tau}^{b} f(v) \frac{\varrho}{\left(\mu_{0}-v\right)^{2}+\varrho^{2}} d \nu \leqq F \varrho \int_{\mu_{0}+\tau}^{b} \frac{1}{\left(\mu_{0}-v\right)^{2}} d v=F \varrho K .
$$

$K$ ist eine von $\varrho$ unabhängige $Z a h l$, so daß

$$
\left|\int_{\mu_{0}+\tau}^{b} f(\nu) \frac{\varrho}{\left(\mu_{0}-v\right)^{2}+\varrho^{2}} d \nu\right| \leqq F \varrho K \leqq \varepsilon \text { sobald } \varrho \leqq \frac{\varepsilon}{F \cdot K}=\delta(\varepsilon) .
$$

Dies bedeutet, daß

$$
\lim _{\varrho \rightarrow 0} \int_{\mu_{0}+\tau}^{b} f(v) \frac{\varrho}{\left(\mu_{0}-v\right)^{2}+\varrho^{2}} d v=0 .
$$

Die beiden Grenzübergänge dürfen also miteinander vertauscht werden.

$$
\lim _{\varrho \rightarrow 0} \int_{\mu_{0}+\tau}^{\infty} f(\nu) \frac{\varrho}{\left(\mu_{0}-v\right)^{2}+\varrho^{2}} d \nu=\lim _{b \rightarrow \infty} \lim _{\varrho \rightarrow 0} \int_{\mu_{0}+\tau}^{b} f(\nu) \frac{\varrho}{\left(\mu_{0}-v\right)^{2}+\varrho^{2}} d \nu=0 .
$$

Somit verschwindet auch das dritte Glied von (4.4):

$$
\lim _{\varrho \rightarrow 0} \int_{\mu_{0}+\tau}^{\infty} f(v) \frac{\varrho}{\left(\mu_{0}-v\right)^{2}+\varrho^{2}} d v=0 \text {. }
$$


Unter Verwendung der Resultate (4.5), (4.8) und (4.9) geht (4.3) über in

$$
\Delta \Gamma\left(p_{0}\right)=2 \pi i f\left(\mu_{0}\right)+2 i R(\tau) .
$$

Bis jetzt war $\tau$ eine beliebige positive Zahl. Diese lassen wir nun gegen 0 konvergieren, wobei nach (4.7) das Restglied $R(\tau)$ gegen 0 strebt. Die Sprungfunktion selber verschwindet aber nicht.

$$
\begin{gathered}
\Delta \Gamma\left(p_{0}\right)=2 \pi i \cdot f\left(\mu_{0}\right)=2 \pi i C_{1} \mu_{0}=-2 \pi C_{1} p_{0} \text { für } \mu_{0} \leqq N \\
\Delta \Gamma\left(p_{0}\right)=-2 \pi C_{1} p_{0} \text { für } N \geqq i p_{0}>0 .
\end{gathered}
$$

Aus diesem Resultat und der Beziehung (4.1) folgt weiter:

$$
\begin{gathered}
\Delta \Gamma\left(p_{0}\right)=\Gamma_{-}\left(p_{0}\right)-\Gamma_{+}\left(p_{0}\right) \\
\Gamma_{+}\left(p_{0}\right)=\Gamma_{-}\left(p_{0}\right)+2 \pi C_{1} p_{0} .
\end{gathered}
$$

Für alle $p$, deren Real- und Imaginärteile negativ sind, führen wir eine neue Funktion ein:

$$
\Gamma^{\prime}(p)=\Gamma(p)-\Delta \Gamma(p) \quad \text { mit } \quad \Delta \Gamma(p)=-2 \pi C_{1} p
$$

Wir wissen, daß $\Gamma(p)$ und $\Delta \Gamma(p)$ in der rechten und linken Hälfte der komplexen Ebene analytisch sind, so daß gilt:

$\Gamma(p)$ analytisch für alle $p$ aus dem 4. Quadranten,

$\Gamma^{\prime}(p)$ analytisch für alle $p$ aus dem 3 . Quadranten.

Außerdem folgt aus (4.11) für alle $p_{0}$ mit $i p_{0} \leqq N$

$$
\begin{aligned}
\lim _{\varrho \rightarrow 0} \Gamma^{\prime}\left(p_{0}-\varrho\right) & =\lim _{\varrho \rightarrow 0} \Gamma\left(p_{0}-\varrho\right)+\lim _{\varrho \rightarrow 0} 2 \pi C_{1}\left(p_{0}-\varrho\right) \\
& =\lim _{\varrho \rightarrow 0} \Gamma\left(p_{0}-\varrho\right)+2 \pi C_{1} p_{0} \\
\lim _{\varrho \rightarrow 0} \Gamma^{\prime}\left(p_{0}-\varrho\right) & =\lim _{\varrho \rightarrow 0} \Gamma\left(p_{0}+\varrho\right) .
\end{aligned}
$$

Somit ist $\Gamma^{\prime}(p)$ für alle $p$ mit $-N \leqq \operatorname{Im}(p)<0$ die analytische Fortsetzung von $\Gamma(p)$ aus dem 4. in den 3. Quadranten ([8], S. 454). Von nun an bedeute $\Gamma(p)$ folgendes (s. Fig. 2):

$$
\Gamma(p)= \begin{cases}\int_{0}^{\infty} \frac{f(v)}{i p-v} d v+2 \pi C_{1} p & \text { falls } p \text { im 3. Quadranten liegt, } \\ \int_{C_{p}^{0}}^{\infty} \frac{f(v)}{i p-v} d v & \text { falls } p \text { auf der negativen ima- } \\ \int_{0}^{\infty} \frac{f(v)}{i p-v} d v & \text { ginären Achse liegt, } \\ & \text { für alle anderen } p, \text { die nicht } \\ & \text { auf der negativen reellen } \\ & \text { Achse liegen. }\end{cases}
$$


Die so definierte Funktion $\Gamma(p)$ ist analytisch für alle $p$, deren Bildpunkte nicht auf der negativen reellen Achse oder auf der imaginären Achse unterhalb - $i N$ liegen.

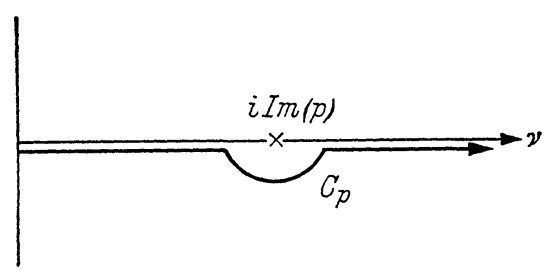

Fig. 2

Falls wir die Wechselwirkung vernachlässigen, ist $f(v)=0$ und damit $\Gamma(p)=0$ oder $=2 \pi C_{1} p$, wobei der Betrag von $2 \pi C_{1} p$ sehr klein ist. Nun soll aber $|\Gamma(p)|$ auch bei Berücksichtigung der Wechselwirkung nie sehr groß werden. Wir nehmen deshalb an, auf einem Kreis $K$ mit Radius $r<v_{0}$ um den Punkt $-i v_{0}$ herum und außerhalb dieses Kreises gelte die Ungleichung

$$
|\Gamma(p)|<\left|i p-v_{0}\right| .
$$

Dann kann die Beziehung $\Gamma(p)=i p-v_{0}$ nur im Innern des Kreises $K$ richtig sein, so daß die Nullstellen der Funktion $i p-v_{0}-\Gamma(p)$ sicher nicht in der oberen Halbebene liegen.

\section{\$ 5. Verschiebung des Integrationsweges}

Bei der Bestimmung der Wahrscheinlichkeitsamplituden mit Hilfe der Formeln (2.5)

$$
\begin{aligned}
a_{0}(t) & =\frac{1}{2 \pi} \lim _{M \rightarrow \infty} \int_{\gamma-i M}^{\gamma+i M} \frac{e^{p t}}{i p-\nu_{0}-\Gamma(p)} d p \\
a(\nu, t) & =\frac{1}{2 \pi} \lim _{M \rightarrow \infty} \int_{\gamma-i M}^{\gamma+i M} \frac{H^{*}(\nu) \cdot e^{p t}}{(i p-v)\left[i p-\nu_{0}-\Gamma(p)\right]} d p
\end{aligned}
$$

liegt der Integrationsweg in der rechten Hälfte der komplexen Ebene, da der Realteil von $p$ positiv sein muß. Wir wollen nun diese Forderung fallen lassen und den Integrationsweg parallel in die linke Halbebene verschieben. Dabei haben wir die singulären Stellen der Integranden zu umgehen.

Der Integrand von $a_{0}(t)$ ist in der ganzen komplexen $p$-Ebene analytisch mit Ausnahme der Punkte $p_{1}$, für die

$$
i p_{1}-v_{0}-\Gamma\left(p_{1}\right)=0
$$

und derjenigen Punkte, in denen $\Gamma(p)$ nicht analytisch ist. Der Integrand von $a(v, t)$ ist für $p=p_{1}$ auch singulär. Daneben hat er im Punkte 
$p_{v}$ mit

$$
i p_{v}-v=0
$$

eine zweite Singularität. Diese liegt auf dem negativen Teil der imaginären Achse, da $p_{\nu}=-i \nu$ mit $\nu \geqq 0$.

Zur Bestimmung der ersten Singularität setzen wir $p_{1}=a_{1}+i b_{1}$. Zunächst nehmen wir an, $p_{1}$ liege nicht im 3. Quadranten. Dann ist nach (4.13)

$$
\begin{aligned}
& i p_{1}-v_{0}-\Gamma\left(p_{1}\right)=i a_{1}-b_{1}-v_{0}-\int_{0}^{\infty} \frac{f(v)}{i a_{1}-b_{1}-v} d v \\
& =-b_{1}-v_{0}+\int_{0}^{\infty} \frac{f(v) \cdot\left(b_{1}+v\right)}{a_{1}^{2}+\left(b_{1}+v\right)^{2}} d v+i a_{1}\left[1+\int_{0}^{\infty} \frac{f(v)}{a_{1}^{2}+\left(b_{1}+v\right)^{2}} d v\right] .
\end{aligned}
$$

Daher kann $i p_{1}-v_{0}-\Gamma\left(p_{1}\right)$ nur verschwinden, falls $a_{1}=0$ und $b_{1}+v_{0}$ $=\int_{0}^{\infty} \frac{f(v)}{b_{1}+\nu} d \nu$. Diese Beziehung läßt sich nur durch negative $b_{1}$ befriedigen, da die Nullstellen von $i p-v_{0}-\Gamma(p)$ nicht in der oberen Halbebene liegen können. Das führt aber auch zu einem Widerspruch, da $b_{1}+v_{0}$ eine reelle Zahl ist, während $\int_{0}^{\infty} \frac{f(v)}{i p-v} d v$ nicht reell ist, da wir um $v=-b_{1}$ herumintegrieren müssen. Somit kann $p_{1}$ nur im 3. Quadranten liegen und es gilt:

$$
\begin{aligned}
i p_{1}-\nu_{0}-\Gamma\left(p_{1}\right)= & i a_{1}-b_{1}-v_{0}-\int_{0}^{\infty} \frac{f(v)}{i a_{1}-b_{1}-v} d v-2 \pi C_{1}\left(a_{1}+i b_{1}\right) \\
& =-b_{1}-v_{0}+\int_{0}^{\infty} \frac{f(v)\left(b_{1}+v\right)}{a_{1}^{2}+\left(b_{1}+v\right)^{2}} d v-2 \pi C_{1} a_{1}+ \\
& +i\left[a_{1}+a_{1} \int_{0}^{\infty} \frac{f(\nu)}{a_{1}^{2}+\left(b_{1}+v\right)^{2}} d \nu-2 \pi C_{1} b_{1}\right] .
\end{aligned}
$$

Der Ausdruck $i p_{1}-v_{0}-\Gamma\left(p_{1}\right)$ wird nun 0 , wenn

$$
\int_{0}^{\infty} \frac{f(v)\left(b_{1}+v\right)}{a_{1}^{2}+\left(b_{1}+v\right)^{2}} d v=b_{1}+v_{0}+2 \pi C_{1} a_{1}
$$

und

$$
a_{1}\left[1+\int_{0}^{\infty} \frac{f(\nu)}{a_{1}^{2}+\left(b_{1}+v\right)^{2}} d v\right]=2 \pi C_{1} b_{1} .
$$

Auf Grund der Annahmen über $\Gamma(p)$ wissen wir, daß die Nullstellen der Funktion $G(p)=i p-v_{0}-\Gamma(p)$ in einem Kreis $K$ mit Radius $r<v_{0}$ 
um den Punkt $-i \nu_{0}$ liegen. Auf $K$ gilt nach (4.14) die Ungleichung $\left|i p-v_{0}\right|>|-\Gamma(p)|$, so daß nach dem Satz von Rouche ([10], S. 88) die Funktionen $i p-v_{0}$ und $i p-v_{0}-\Gamma(p)$ in der Anzahl der Nullstellen übereinstimmen. Somit gibt es immer genau ein $p_{1}$, das im 3. Quadranten in der Umgebung von $-i v_{0}$ liegt und der Beziehung (5.1) genügt.

Nun ändern wir den Integrationsweg in (2.5), wobei wir darauf zu achten haben, daß die komplexe Ebene von $-i N$ bis $-i \infty$ und von $-\infty$ bis 0 aufgeschlitzt ist.

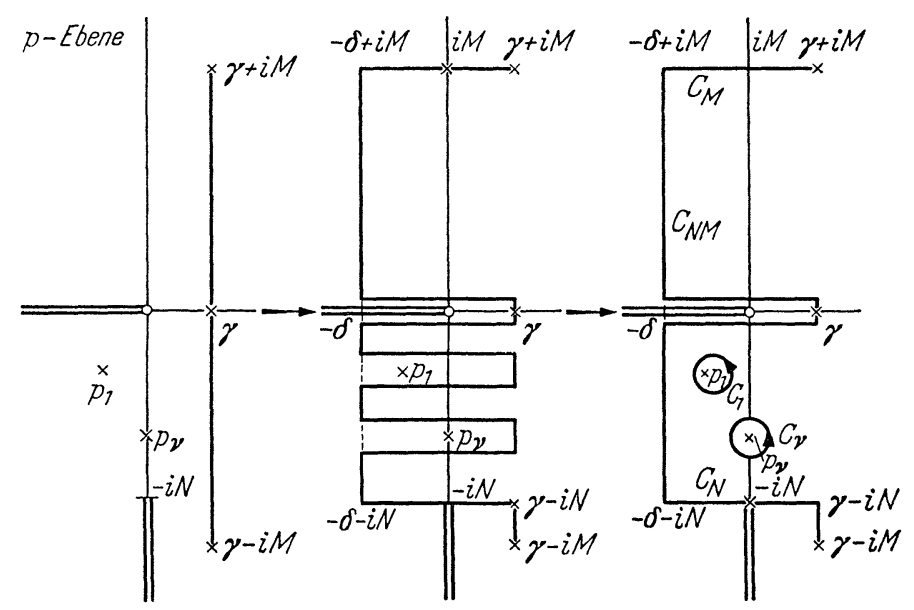

Fig. 3

Wir erhalten dadurch folgende Beiträge zu den Integralen

$\frac{1}{2 \pi} \int_{\gamma-i M}^{\gamma+i M} \frac{e^{\nu t}}{i p-v_{0}-\Gamma(p)} d p$ und $\frac{1}{2 \pi} \int_{\gamma-i M}^{\gamma+i M} \frac{H^{*}(\nu) \cdot e^{p t}}{(i p-\nu)\left[i p-v_{0}-\Gamma(p)\right]} d p$.

1) Integral von $\gamma-i M$ bis $-\delta-i N$ über $c_{N}$,

2) Integral von $-\delta-i N$ bis $-\delta+i M$ über $c_{N M}$,

3) Integral von $-\delta+i M$ bis $\gamma+i M$ über $c_{M}$,

4) Integral über $c_{1}$ um den Pol $p_{1}$ herum,

5) Integral über $c_{v}$ um den Pol $p_{v}$ herum.

Einige dieser Teilintegrale können wir direkt berechnen. Es ist beispielsweise

$$
\frac{1}{2 \pi} \oint_{C_{\nu}} \frac{H^{*}(v) \cdot e^{p t}}{(i p-v)\left[i p-v_{0}-\Gamma(p)\right]} d p=\frac{H^{*}(v)}{2 \pi i} \oint_{C_{v}} \frac{e^{p t} / i p-v_{0}-\Gamma(p)}{p-(-i v)} d p
$$

Nach der Integralformel von Cauchy ist

$$
\frac{1}{2 \pi i} \oint_{C_{v}} \frac{e^{p t} / i p-v_{0}-\Gamma(p)}{p-(-i v)} d p=\frac{e^{-i v t}}{i(-i v)-v_{0}-\Gamma(-i v)} .
$$


Somit finden wir

$$
\frac{1}{2 \pi} \oint_{C_{\nu}} \frac{H^{*}(\nu) e^{p t}}{(i p-\nu)\left[i p-\nu_{0}-\Gamma(p)\right]} d p=\frac{H^{*}(v) \cdot e^{-i \nu t}}{\nu-v_{0}-\Gamma(-i v)} .
$$

Nun berechnen wir das Glied $\frac{1}{2 \pi} \oint_{C_{1}} \frac{e^{p t} d p}{i p-v_{0}-\Gamma(p)}$. Der Nenner $G(p)$ $=i p-v_{0}-\Gamma(p)$ ist eine analytische Funktion von $p$, die in der Umgebung von $p_{1}$ in eine Potenzreihe entwickelt wird.

$$
G(p)=G\left(p_{1}\right)+\left(p-p_{1}\right) G^{\prime}\left(p_{1}\right)+\cdots .
$$

Nach (5.1) ist $p_{1}$ eine Nullstelle von $G(p)$. Um zu zeigen, daß diese von der 1. Ordnung ist, berechnen wir $G^{\prime}(p)$ für solche $p$, deren Real- und Imaginärteile negativ sind.

$$
G^{\prime}(p)=i-\Gamma^{\prime}(p)=i-\frac{d}{d p} \int_{0}^{\infty} \frac{f(v)}{i p-\nu} d v-2 \pi C_{1} .
$$

Das Integral darf unter dem Integrationszeichen differenziert werden, da das Integral der Ableitung des Integranden gleichmäßig existiert ([9], Bd. III, S. 299). Es ist nämlich für $p=x+i y$ und $y>-N$

$$
\left|\int_{\mu_{1}}^{\mu_{2}}-i \cdot \frac{f(v)}{(i p-v)^{2}} d \nu\right| \leqq \int_{\mu_{1}}^{\mu_{2}} \frac{f(v)}{x^{2}+(y+v)^{2}} d \nu \leqq \int_{\mu_{1}}^{\mu_{2}} \frac{f(v)}{(y+v)^{2}} d \nu .
$$

Es ist $v+y>v-N>0$, falls wir $\mu_{1}>N$ wählen. Dann erhalten wir

$$
\begin{array}{r}
\int_{\mu_{1}}^{\mu_{2}} \frac{f(\nu)}{(y+\nu)^{2}} d \nu \leqq \int_{\mu_{1}}^{\mu_{2}} \frac{C \cdot \nu^{-\alpha}}{(\nu-N)^{2}} d \nu \leqq C \int_{\mu_{1}}^{\mu_{2}}(\nu-N)^{-(\alpha+2)} d \nu<C\left(\mu_{1}-N\right)^{-(\alpha+1)} \leqq \varepsilon \\
\text { sobald } \mu_{1} \geqq N+\left(\frac{C}{\varepsilon}\right)^{\frac{1}{\alpha+1}} .
\end{array}
$$

Somit ist

$$
G^{\prime}(p)=i+i \int_{0}^{\infty} \frac{f(\nu)}{(i p-v)^{2}} d \nu-2 \pi C_{1}
$$

Um nun anzugeben, daß Real- und Imaginärteil negativ sein sollen, setzen wir $p=-a-i b$. Dann ist

$$
\begin{gathered}
G^{\prime}(p)=i+i \int_{0}^{\infty} \frac{f(\nu)}{(b-v-i a)^{2}} d \nu-2 \pi C_{1} \\
G^{\prime}(p)=-2 a \int_{0}^{\infty} \frac{f(v)(b-v)}{\left[(b-v)^{2}+a^{2}\right]^{2}} d \nu-2 \pi C_{1}+ \\
+i\left\{1+\int_{0}^{\infty} \frac{f(v)\left[(b-v)^{2}-a^{2}\right]}{\left[(b-v)^{2}+a^{2}\right]^{2}} d \nu\right\} .
\end{gathered}
$$


Unter Benutzung der Beziehung (5.4) untersuchen wir den Imaginärteil.

$$
\begin{aligned}
& 1+\int_{0}^{\infty} \frac{f(v)\left[(b-v)^{2}-a^{2}\right]}{\left[(b-v)^{2}+a^{2}\right]^{2}} d v=1+\int_{0}^{\infty} \frac{f(v)\left[(b-v)^{2}+a^{2}\right]}{\left[(b-v)^{2}+a^{2}\right]^{2}} d v- \\
& \quad-2 a^{2} \int_{0}^{\infty} \frac{f(v) d v}{\left[(b-v)^{2}+a^{2}\right]^{2}}=\frac{2 \pi C_{1} b}{a}-2 a^{2} \int_{0}^{\infty} \frac{f(v)}{\left[(b-v)^{2}+a^{2}\right]^{2}} d v .
\end{aligned}
$$

Der größte Beitrag zum letzten Integral kommt aus der Umgebung von $\boldsymbol{v}=b$, da $f(v)$ stetig und nur langsam veränderlich ist.

$$
\begin{aligned}
\int_{0}^{\infty} \frac{f(v)}{\left[(b-v)^{2}+a^{2}\right]^{2}} d v & \approx f(b) \int_{0}^{\infty} \frac{d v}{\left[(b-v)^{2}+a^{2}\right]^{2}} \\
= & \frac{f(b)}{2 a^{2}}\left[\frac{v-b}{(v-b)^{2}+a^{2}}+\frac{1}{a} \operatorname{arctg} \frac{v-b}{a}\right]_{0}^{\infty} \\
& =\frac{f(b)}{2 a^{2}}\left[\frac{b}{b^{2}+a^{2}}+\frac{\pi}{2 a}+\frac{1}{a} \operatorname{arctg} \frac{b}{a}\right]<\frac{f(b)}{2 a^{2}}\left[\frac{b}{b^{2}+a^{2}}+\frac{\pi}{a}\right] .
\end{aligned}
$$

Also ist

$$
\begin{aligned}
1+\int_{0}^{\infty} \frac{f(v)\left[(b-v)^{2}-a^{2}\right]}{\left[(b-v)^{2}+a^{2}\right]^{2}} & d v>\frac{2 \pi f(b)}{a}-\frac{b \cdot f(b)}{b^{2}+a^{2}}-\frac{f(b) \pi}{a} \\
& =\frac{\pi f(b)}{a}-\frac{b \cdot f(b)}{b^{2}+a^{2}}>\frac{f(b)}{a\left(a^{2}+b^{2}\right)}\left(a^{2}+b^{2}-a b\right)>0 .
\end{aligned}
$$

Aus dieser Abschätzung geht hervor, daß der Imaginärteil von $G^{\prime}\left(p_{1}\right)$ positiv ist, daß also $G^{\prime}\left(p_{1}\right)$ nicht 0 sein kann. Somit haben wir nachgewiesen, daß jedes $p_{1}$ aus dem dritten Quadranten eine Nullstelle 1. Ordnung von $G(p)$ sein kann. Daher können wir schreiben:

$$
\frac{e^{p t}}{i p-\nu_{0}-\Gamma(p)}=\frac{e^{p t}}{\left(p-p_{1}\right) \cdot G^{\prime}\left(p_{1}\right)+\cdots}
$$

und die Integralformel von $\mathrm{CAUCHY}_{\mathrm{A}}$ anwenden.

Es ist also

$$
\oint_{C_{1}} \frac{e^{p t}}{\left(p-p_{1}\right) \cdot G^{\prime}\left(p_{1}\right)+\cdots} d p=2 \pi i \frac{e^{p_{1} t}}{G^{\prime}\left(p_{1}\right)} \text {. }
$$

$$
\frac{1}{2 \pi} \oint_{C_{1}} \frac{e^{p t}}{i p-v_{0}-\Gamma(p)} d p=\frac{i \cdot e^{p_{1} \ell}}{i-\Gamma^{\prime}\left(p_{1}\right)}=\frac{e^{p_{1} \ell}}{1+i \Gamma^{\prime}\left(p_{1}\right)}
$$

Die vorangehenden Überlegungen gelten auch für $\frac{1}{2 \pi} \oint_{C_{1}} \frac{e^{p t} H^{*}(v) d p}{(i p-v)\left[i p-v_{0}-\Gamma(p)\right]}$, da wir wissen, daß $i p_{1}-\boldsymbol{\nu} \neq 0$. Somit ist

$$
\begin{aligned}
\frac{1}{2 \pi} \oint_{C_{1}} \frac{H^{*}(v) \cdot e^{p t}}{(i p-v)\left[i p-v_{0}-\Gamma(p)\right]} d p & =\frac{i \cdot H^{*}(v) e^{p_{1} \ell}}{\left(i p_{1}-v\right)\left[i-\Gamma^{\prime}\left(p_{1}\right)\right]} \\
& =\frac{H^{*}(\nu) e^{p_{1} \iota}}{\left(i p_{1}-v\right)\left[1+i \Gamma^{\prime}\left(p_{1}\right)\right]} .
\end{aligned}
$$


Für die Wahl von $\delta$ haben wir nur die Einschränkung, daß der Integrationsweg links von den Singularitäten liegen muß. Wir können daher $\delta$ gegen Unendlich gehen lassen und die Grenzwerte derjenigen Glieder von (5.5) berechnen, die von $\delta$ abhängen.

Als erstes untersuchen wir die Grenzwerte von $\int_{C_{N} M} \frac{e^{p t}}{i p-v_{0}-\Gamma(p)} d p$ und $\int_{C_{N} M} \frac{e^{p t} d p}{(i p-v)\left[i p-v_{0}-\Gamma(p)\right]}$. Dazu zerlegen wir die Integrale:

$$
\begin{aligned}
\int_{C_{N M}} \frac{e^{p t}}{i p-v_{0}-\Gamma(p)} d p=\int_{-\delta-i N}^{-\delta-i \beta} \frac{e^{p t}}{i p-v_{0}-\Gamma(p)} d p+ \\
\quad+\int_{-\delta-i \beta}^{-\delta+i \beta} \frac{e^{p t}}{i p-v_{0}-\Gamma(p)} d p+\int_{-\delta+i \beta}^{-\delta+i M} \frac{e^{p t}}{i p-\nu_{0}-\Gamma(p)} d p .
\end{aligned}
$$

Nun ist für $p=-\delta-i y$ und $d p=-i d y$

$$
\begin{aligned}
& \lim _{\delta \rightarrow \infty} \int_{-\delta-i N}^{-\delta-i \beta} \frac{e^{p t}}{i p-v_{0}-\Gamma(p)} d p \\
& \quad=\lim _{\delta \rightarrow \infty} e^{-\delta t} \cdot \lim _{\delta \rightarrow \infty}\left\{-i \int_{N}^{\beta} \frac{e^{-i \nu t}}{y-v_{0}-i \delta-\Gamma(-\delta-i y)} d y\right\} .
\end{aligned}
$$

Da der erste Faktor gegen 0 strebt, genügt es zu zeigen, daß der zweite beschränkt ist. Nun gilt

$$
\begin{aligned}
y-v_{0}-i \delta-\Gamma(-\delta-i y)= & y-v_{0}-i \delta+\int_{0}^{\infty} \frac{f(v)}{v-y+i \delta} d \nu+2 \pi C_{1}(\delta+i y) \\
= & \left(2 \pi C_{1}-i\right) \delta+y-v_{0}+2 \pi C_{1} i y+ \\
& +\int_{0}^{\infty} \frac{f(v)}{v-y+i \delta} d v .
\end{aligned}
$$

Der letzte Summand verschwindet für große $\delta$ gleichmäßig in $y$, denn es ist

$$
\left|\int_{0}^{\infty} \frac{f(v)}{\nu-y+i \delta} d \nu\right| \leqq \int_{0}^{\infty} \frac{f(v)}{\sqrt{(\nu-y)^{2}+\delta^{2}}} d \nu \leqq \frac{1}{\delta} \int_{0}^{\infty} f(v) d \nu .
$$

Somit ist für $\delta \geqq \delta_{0}$

Hieraus folgt

$$
\left|y-v_{0}-i \delta-\Gamma(-\delta-i y)\right| \geqq A .
$$

$$
\left|-i \int_{N}^{\beta} \frac{e^{-i y t}}{y-v_{0}-i \delta-\Gamma(-\delta-i y)} d y\right| \leqq \int_{\beta}^{N} \frac{1}{A} d y=C
$$

24 Commun. math. Phys., Vol. 2 
für genügend große $\delta$. Das Integral von $-\delta-i N$ bis $-\delta-i \beta$ konvergiert daher gegen 0 , wenn $\delta$ gegen Unendlich strebt.

Die gleichen Überlegungen gelten auch für das Integral von $-\delta+i \beta$ bis $-\delta+i M$. Folglich ist

$$
\lim _{\delta \rightarrow \infty} \int_{C_{N M}} \frac{e^{p t}}{i p-v_{0}-\Gamma(p)} d p=\int_{C_{\beta}} \frac{e^{p t}}{i p-v_{0}-\Gamma(p)} d p .
$$

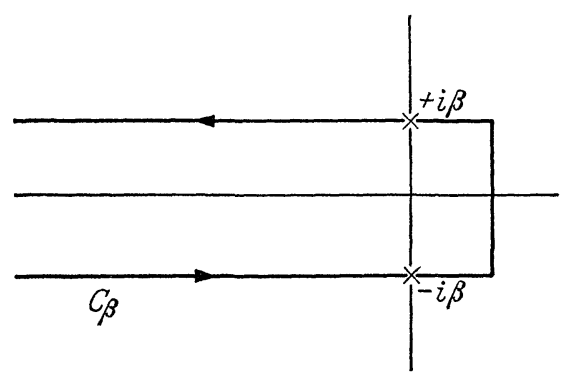

Fig. 4

Analog beweist man auch, daß

$\lim _{\delta \rightarrow \infty} \int_{C_{N M}} \frac{e^{p t}}{(i p-v)\left[i p-v_{0}-\Gamma(p)\right]} d p=\int_{C_{\beta}} \frac{e^{p t}}{(i p-v)\left[i p-v_{0}-\Gamma(p)\right]} d p$.

Durch ähnliche Abschätzungen wie vorhin können wir auch zeigen, daß die Grenzwerte

$\lim _{\delta \rightarrow \infty} \int_{C_{M}} \frac{e^{p t}}{i p-v_{0}-\Gamma(p)} d p$ und $\lim _{\delta \rightarrow \infty} \int_{C_{M}} \frac{e^{p t}}{(i p-v)\left[i p-v_{0}-\bar{\Gamma}(p)\right]} d p$

gleichmäßig in $M$ existieren.

Um nun aus der Zerlegung (5.5) die Ausdrücke für $a_{0}(t)$ und $a(v, t)$ zu erhalten, lassen wir auch noch $M$ gegen Unendlich konvergieren. Unter Verwendung der Beziehungen (5.5), (5.6), (5.9)-(5.12) erhalten wir:

$$
\begin{aligned}
a_{0}(t)= & \frac{1}{2 \pi} \lim _{M \rightarrow \infty} \lim _{\delta \rightarrow \infty} \int_{C_{N}} \frac{e^{p t}}{i p-v_{0}-\Gamma(p)} d p+ \\
& +\frac{1}{2 \pi} \lim _{M \rightarrow \infty} \lim _{\delta \rightarrow \infty} \int_{C_{M}} \frac{e^{p t}}{i p-v_{0}-\Gamma(p)} d p+ \\
& +\frac{1}{2 \pi} \int_{C_{\beta}} \frac{e^{p t}}{i p-v_{0}-\Gamma(p)} d p+\frac{e^{p_{1} t}}{1+i \Gamma^{\prime}\left(p_{1}\right)},
\end{aligned}
$$




$$
\begin{aligned}
a(\nu, t)= & \frac{1}{2 \pi} \lim _{M \rightarrow \infty} \lim _{\delta \rightarrow \infty} \int_{C_{N}} \frac{H^{*}(v) \cdot e^{p t}}{(i p-v)\left[i p-v_{0}-\Gamma(p)\right]} d p+ \\
& +\frac{1}{2 \pi} \lim _{M \rightarrow \infty} \lim _{\delta \rightarrow \infty} \int_{C_{M}} \frac{H^{*}(v) \cdot e^{p t}}{(i p-v)\left[i p-v_{0}-\Gamma(p)\right]} d p+ \\
& +\frac{1}{2 \pi} \int_{C_{\beta}} \frac{H^{*}(\nu) \cdot e^{p t}}{(i p-v)\left[i p-v_{0}-\Gamma(p)\right]} d p+ \\
& +\frac{H^{*}(v) \cdot e^{p_{1} t}}{\left(i p_{1}-v\right)\left[1+i \Gamma^{\prime}\left(p_{1}\right)\right]}+\frac{H^{*}(v) \cdot e^{-i v t}}{v-v_{0}-\Gamma(-i v)} .
\end{aligned}
$$

Je das erste Glied von (5.14) und (5.15) hängt von der Art des Abschneidens der Funktion $f(v)$ ab. Wir untersuchen daher diese Grenzwerte nicht und setzen dafür

$$
\begin{aligned}
& A=\frac{1}{2 \pi} \lim _{M \rightarrow \infty} \lim _{\delta \rightarrow \infty} \int_{C_{N}} \frac{e^{p t}}{i p-v_{0}-\Gamma(p)} d p \\
& B=\frac{1}{2 \pi} \lim _{M \rightarrow \infty} \lim _{\delta \rightarrow \infty} \int_{C_{N}} \frac{H^{*}(v) \cdot e^{p t}}{(i p-v)\left[i p-v_{0}-\Gamma(p)\right]} d p .
\end{aligned}
$$

Wir werden nun noch zeigen, daß die Grenzwerte

und

$$
\lim _{M \rightarrow \infty} \lim _{\delta \rightarrow \infty} \int_{C_{M}} \frac{e^{p t}}{i p-v_{0}-\Gamma(p)} d p
$$

$$
\lim _{M \rightarrow \infty} \lim _{\delta \rightarrow \infty} \int_{C_{M}} \frac{e^{p t}}{(i p-v)\left[i p-v_{0}-\Gamma(p)\right]} d p
$$

verschwinden. $\mathrm{Zu}$ diesem $\mathrm{Zwecke}$ berechnen wir für beide Integrale den Grenzwert für $M \rightarrow \infty$, wobei wir $p=x+i M$ setzen.

$$
\begin{aligned}
\int_{-\delta+i M}^{\gamma+i M} \frac{e^{p t}}{i p-v_{0}-\Gamma(p)} d p \mid \leqq \int_{-\delta}^{\gamma} \frac{\left|e^{x t} \cdot e^{i M t}\right|}{\left|i x-M-v_{0}-\Gamma(x+i M)\right|} d x \leqq \\
\leqq \int_{-\delta}^{\gamma} \frac{e^{x t}}{\left|M+v_{0}+\operatorname{Re} \Gamma(x+i M)\right|} d x .
\end{aligned}
$$

Nun ist

$$
\begin{aligned}
M+v_{0}+\operatorname{Re} \Gamma & (x+i M)=M+v_{0}-\int_{0}^{\infty} \frac{f(v)(M+v)}{(M+v)^{2}+x^{2}} d v>M+v_{0}- \\
& -\int_{0}^{\infty} \frac{f(v)}{M+v} d v>M-\int_{0}^{\infty} \frac{f(v)}{1+v} d v>0 \text { falls } M>M_{0} \\
& =\max \left[1, \int_{0}^{\infty} \frac{f(v)}{1+v} d v\right] .
\end{aligned}
$$


Für die weitere Abschätzung sei $M>M_{0}$.

$$
\begin{aligned}
\int_{-\delta}^{\gamma} \frac{e^{x t}}{\left|M+v_{0}+\operatorname{Re} \Gamma(x+i M)\right|} d x<\int_{-\infty}^{\gamma} \frac{e^{x t}}{M-\int_{0}^{\infty} \frac{f(\nu)}{1+\nu} d \nu} d x< \\
<\frac{e^{\gamma t}}{t\left(M-M_{0}\right)} \leqq \varepsilon \text { sobald } M \geqq M_{0}+\frac{e^{\gamma t}}{\varepsilon t} .
\end{aligned}
$$

Ähnlich verläuft auch die Abschätzung des zweiten Integrals. Folglich konvergieren die Integrale

$$
\int_{-\delta+i M}^{\gamma+i M} \frac{e^{p t}}{i p-v_{0}-\Gamma(p)} d p \text { und } \int_{-\delta+i M}^{\gamma+i M} \frac{e^{p t}}{(i p-v)\left[i p-v_{0}-\Gamma(p)\right]} d p
$$

für $M \rightarrow \infty$ gleichmäßig in $\delta$ gegen 0 . Da der Grenzwert dieser Integrale auch für $\delta \rightarrow \infty$ existiert [vgl. (5.13)], können wir die Grenzübergänge $M \rightarrow \infty$ und $\delta \rightarrow \infty$ vertauschen ([9], Bd. II, S. 146). Somit erhalten wir:

$$
\begin{aligned}
\lim _{M \rightarrow \infty} \lim _{\delta \rightarrow \infty} \int_{-\delta+i M}^{\gamma+i M} \frac{e^{p t}}{i p-v_{0}-\Gamma(p)} d p \\
=\lim _{M \rightarrow \infty} \lim _{\delta \rightarrow \infty} \int_{-\delta+i M}^{\gamma+i M} \frac{e^{p t}}{(i p-\nu)\left[i p-v_{0}-\Gamma(p)\right]} d p=0 .
\end{aligned}
$$

Unter Verwendung von (5.16) gehen somit die Darstellungen (5.14) und (5.15) der Wahrscheinlichkeitsamplituden über in:

$$
\begin{gathered}
a_{0}(t)=A+\frac{e^{p_{1} \iota}}{1+i \Gamma^{\prime}\left(p_{1}\right)}+\frac{1}{2 \pi} \int_{C_{\beta}} \frac{e^{p t}}{i p-v_{0}-\Gamma(p)} d p \\
a(v, t)=B+\frac{H^{*}(v) e^{p_{1}} t}{\left(i p_{1}-v\right)\left[1+i \Gamma^{\prime}\left(p_{1}\right)\right]}+\frac{H^{*}(v) e^{-i v t}}{v-v_{0}-\Gamma(-i v)}+ \\
\quad+\frac{1}{2 \pi} \int_{C_{\beta}} \frac{H^{*}(v) e^{p t}}{(i p-v)\left[i p-v_{0}-\Gamma(p)\right]} d p .
\end{gathered}
$$

\section{§ 6. Berechnung der Wahrscheinlichkeiten}

Zur Berechnung der Wahrscheinlichkeiten auf Grund der Formeln (5.17) und (5.18) berücksichtigen wir die Beiträge $A$ und $B$ nicht, da sie ja von der Art des Abschneidens für $v \geqq N$ abhängen. Auch vernachlässigen wir zunächst die Glieder, die von der Integration um die negative reelle Achse herrühren, so daß sich die Berechnung der Wahrscheinlichkeiten $\left|a_{0}(t)\right|^{2}$ und $|a(v, t)|^{2}$ vereinfacht. Es ist dann für $p_{1}=a_{1}+i b_{1}$

$$
\begin{gathered}
\left|a_{0}(t)\right|^{2}=\frac{e^{\left(a_{1}+i b_{1}\right) t \cdot e\left(a_{1}-i b_{1}\right) t}}{\left[1+i \Gamma^{\prime}\left(p_{1}\right)\right] \cdot\left[1-i \Gamma^{\prime}\left(p_{1}\right)^{*}\right]}=\frac{e^{2 a_{1} t}}{K\left(p_{1}\right)} \\
\frac{d\left|a_{0}(t)\right|^{2}}{d t}=\frac{2 a_{1} \cdot e^{2 a_{1} t}}{K\left(p_{1}\right)} .
\end{gathered}
$$


$\left|a_{0}(t)\right|^{2}$ ist die Wahrscheinlichkeit dafür, daß zur Zeit $t$ das Atomelektron angeregt ist, und daß noch kein Lichtquant existiert. $\frac{d\left|a_{0}(t)\right|^{2}}{d t}$ gibt die Änderung dieser Wahrscheinlichkeit pro Sekunde an, ist also die gesamte Übergangswahrscheinlichkeit pro Sekunde zur Zeit $t$.

Da $a_{1}$ und $b_{1}$ negativ sind, setzen wir $a_{1}=-a$ und $b_{1}=-b$.

$$
\left|a_{0}(t)\right|^{2}=\frac{e^{-2 a t}}{K\left(p_{1}\right)} ; \frac{d\left|a_{0}(t)\right|^{2}}{d t}=-2 a \cdot \frac{e^{-2 a t}}{K\left(p_{1}\right)} .
$$

Die Wahrscheinlichkeit, daß sich das Atom zu einem späteren Zeitpunkt noch im Anfangszustand befindet, nimmt exponentiell ab. Sie ist am größten für $t=0$ und sinkt auf $\frac{1}{e}$ dieses Wertes für $T=\frac{1}{2 a}$. Für sehr große Zeiten ist $\left|a_{0}(t)\right|^{2}=0$, das Atom ist wieder im Grundzustand. Die Zahl $a$ ist also ein Maß für die Beständigkeit des angeregten Zustandes.

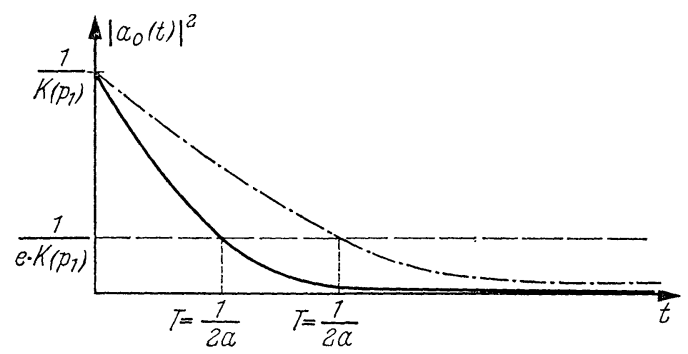

Fig. 5. - - - - für kleine Werte von a, - für große Werte von a.

Nun berechnen wir nach (5.18) die Wahrscheinlichkeit, daß bis zur Zeit $t$ Licht der Frequenz $v$ ausgestrahlt wird.

$$
\begin{aligned}
|a(v, t)|^{2} & =|H(v)|^{2}\left\{\frac{e^{-2 a t}}{K\left(p_{1}\right) \cdot\left[(\nu-b)^{2}+a^{2}\right]}+\right. \\
& +\frac{1}{\left[\nu-v_{0}-\Gamma(-i v)\right] \cdot\left[v-v_{0}-\Gamma(-i v)^{*}\right]}+ \\
& +\frac{e^{-a t} \cdot e^{i(\nu-b) t}}{\left[1+i \Gamma^{\prime}\left(p_{1}\right)\right] \cdot(-i a+b-v)\left[\nu-v_{0}-\Gamma(-i v)^{*}\right]}+ \\
& \left.+\frac{e^{-a t} \cdot e^{-i(v-b) t}}{\left[1-i \Gamma^{\prime}\left(p_{1}\right)^{*}\right](i a+b-v)\left[v-v_{0}-\Gamma(-i v)\right]}\right\} .
\end{aligned}
$$

Wir untersuchen $|a(v, t)|^{2}$ nur für Zeiten, die groß sind gegenüber der Lebensdauer des angeregten Zustandes. Dadurch verschwinden das erste, dritte und vierte Glied. Außerdem setzen wir

$$
\Gamma(-i \nu)=\int_{C_{p}} \frac{f(\mu)}{i(-i v)-\mu} d \mu=\int_{C_{p}} \frac{f(\mu)}{\nu-\mu} d \mu=\gamma(v) .
$$


Nach (4.11) ist dann

$$
\gamma(v)-\gamma^{*}(v)=-2 \pi i C_{1} v=-2 \pi i f(v),
$$

so daß wir erhalten

$$
\operatorname{Im} \gamma(v)=-\pi f(v) .
$$

Für sehr große Zeiten gilt somit

$$
\begin{aligned}
|a(\nu, \infty)|^{2} & =\frac{|H(v)|^{2}}{\left[\nu-v_{0}-\gamma(v)\right] \cdot\left[\nu-v_{0}-\gamma^{*}(v)\right]} \\
& =\frac{|H(v)|^{2}}{\left[\nu-v_{0}-\operatorname{Re} \gamma(v)\right]^{2}+[\operatorname{Im} \gamma(v)]^{2}} \\
|a(\nu, \infty)|^{2} & =\frac{|H(v)|^{2}}{\left[\nu-v_{0}-\operatorname{Re} \gamma(v)\right]^{2}+\pi^{2} f(v)^{2}} .
\end{aligned}
$$

Zur Bestimmung der Intensitätsverteilung des ausgestrahlten Lichtes addieren wir alle Emissionswahrscheinlichkeiten für die Frequenzen zwischen $v$ und $v+d v$. Diese gesamte Emissionswahrscheinlichkeit bezeichnen wir mit $P_{\infty}(v) d v$.

$$
P_{\infty}(v) d v=\int_{0}^{4 \pi} \frac{|H(v, \Omega)|^{2}}{\left[v-v_{0}-\operatorname{Re} \gamma(v)\right]^{2}+\pi^{2} f(v)^{2}} \varrho(v) d v d \Omega .
$$

Unter Benutzung der Bezeichnungen aus $\S 2$ erhalten wir dann:

$$
P_{\infty}(\nu) d \nu=\frac{f(v) d v}{\left[\nu-v_{0}-\operatorname{Re} \gamma(\nu)\right]^{2}+\pi^{2} f(v)^{2}} .
$$

Für die Intensitätsverteilung $I(v)$, die proportional zu $P_{\infty}(v)$ ist, gilt daher folgende Formel:

$$
I(v)=I_{0} \frac{f(v)}{\left[v-v_{0}-\operatorname{Re} \gamma(v)\right]^{2}+\pi^{2} f(v)^{2}} .
$$

Die Funktion $f(v)$ ist stetig und nur langsam veränderlich, so daß die Intensität für $v \approx v_{1}$ mit

$$
v_{1}-v_{0}-\operatorname{Re} \gamma\left(v_{1}\right)=0
$$

das Maximum erreicht. Dieses liegt also nicht genau bei $v_{0}$, es gibt eine Niveauverschiebung um $\operatorname{Re} \gamma\left(v_{1}\right)$. Diese Verschiebung werden wir nun berechnen.

Nach (2.2) und (6.2) ist

$\gamma(\nu)=C_{1}\left[\int_{0}^{\nu-\varepsilon} \frac{\mu}{\nu-\mu} d \mu+\int_{\nu-\varepsilon}^{\nu+\varepsilon} \frac{\mu}{\nu-\mu} d \mu-\int_{\nu+\varepsilon}^{N} \frac{\mu}{\mu-\nu} d \mu\right]-C \int_{N}^{\infty} \frac{\mu^{-\alpha}}{\mu-\nu} d \mu$.

Diese Integrale können berechnet werden.

$$
\begin{aligned}
& \int_{0}^{\nu-\varepsilon} \frac{\mu}{\nu-\mu} d \mu=[-\mu-\nu \ln (\nu-\mu)]_{0}^{\nu-\varepsilon}=\varepsilon-\nu-\nu \ln \frac{\varepsilon}{\nu} . \\
& \int_{\nu+\varepsilon}^{N} \frac{\mu}{\mu-\nu} d \mu=[\mu+\nu \ln (\mu-\nu)]_{\nu+\varepsilon}^{N}=N-\nu-\varepsilon+\nu \ln \frac{N-\nu}{\varepsilon} .
\end{aligned}
$$


Durch Substitution $\mu=v+\varepsilon e^{i \varphi}$ erhalten wir:

$\int_{C_{p}}^{\nu+\varepsilon} \frac{\mu}{\nu-\mu} d \mu=\int_{\pi}^{2 \pi} \frac{\nu+\varepsilon e^{i \varphi}}{-\varepsilon e^{i \varphi}} \varepsilon i e^{i \varphi} d \varphi=-i\left[\nu \pi+\frac{\varepsilon}{i^{2}}\right]=-2 \varepsilon-i \nu \pi$.

Zur Berechnung des vierten Integrals setzen wir $\alpha=z$.

$$
\int_{N}^{\infty} \frac{1}{\mu^{2}(\mu-v)} d \mu=\left[\frac{1}{v \mu}+\frac{1}{v^{2}} \ln \frac{\mu-v}{\mu}\right]_{N}^{\infty}=-\left[\frac{1}{v N}+\frac{1}{v^{2}} \ln \frac{N-v}{N}\right] .
$$

Da $\frac{v}{N}<1$ ist, können wir näherungsweise $\ln \frac{N-v}{N}$ durch $-\frac{v}{N}$ ersetzen, so daß das vierte Integral keinen Beitrag liefert. Somit ist

$$
\gamma(\nu)=-C_{1}\left[N+v \ln \frac{N-v}{\nu}\right]-i C_{1} \nu \pi
$$

Die Frequenz $v_{1}$ muß daher der Gleichung

genügen.

$$
v_{0}-v_{1}=C_{1}\left[N+v_{1} \cdot \ln \frac{N-v_{1}}{v_{1}}\right]
$$

Wir berechnen auch die Frequenz $v_{2}$, für welche die Intensität noch die Hälfte der maximalen beträgt.

$$
\begin{gathered}
I\left(\nu_{2}\right)=I_{0} \frac{f\left(v_{0}\right)}{\left[\nu_{2}-v_{0}-\operatorname{Re} \gamma\left(v_{2}\right)\right]^{2}+\pi^{2} f\left(v_{0}\right)^{2}}=\frac{I_{0}}{2} \cdot \frac{f\left(v_{0}\right)}{\pi^{2} f\left(v_{0}\right)^{2}} \\
\left|v_{2}-v_{0}-\operatorname{Re} \gamma\left(v_{2}\right)\right|=\pi f\left(v_{0}\right) .
\end{gathered}
$$

Die Differenz $\Delta v=\left|v_{2}-v_{1}\right|$ ist ein Maß für die Breite der Spektrallinie. Wir werden nun diese Größe, die Halbwertsbreite, berechnen. $\nu_{2}-\nu_{0}-\operatorname{Re} \gamma\left(\nu_{2}\right)=\nu_{2}-\nu_{1}-\operatorname{Re} \gamma\left(\nu_{2}\right)+\operatorname{Re} \gamma\left(\nu_{1}\right)+\nu_{1}-\nu_{0}-\operatorname{Re} \gamma\left(\nu_{1}\right)$. Nach (6.7) ist

$$
\operatorname{Re}\left[\gamma\left(\nu_{1}\right)-\gamma\left(v_{2}\right)\right]=C_{1}\left[\nu_{2} \ln \frac{N-v_{2}}{\nu_{2}}-v_{1} \ln \frac{N-\nu_{1}}{\nu_{1}}\right] .
$$

Wir können teilweise $\nu_{2}$ durch $\nu_{1}$ ersetzen, da diese Frequenzen beinahe gleich sind.

$$
\operatorname{Re}\left[\gamma\left(\nu_{1}\right)-\gamma\left(\nu_{2}\right)\right]=C_{1}\left(\nu_{2}-v_{1}\right) \ln \frac{N-v_{1}}{v_{1}}
$$

Somit erhalten wir unter Berücksichtigung von (6.6)

$$
v_{2}-v_{0}-\operatorname{Re} \gamma\left(v_{2}\right)=\left(v_{2}-v_{1}\right)\left[1+C_{1} \ln \frac{N-v_{1}}{v_{1}}\right] \text {. }
$$

Durch Vergleich mit (6.9) erhalten wir folgenden Ausdruck für die Halbwertsbreite:

$$
\Delta v=\frac{\pi f\left(v_{0}\right)}{1+C_{1} \cdot \ln \frac{N-v_{1}}{v_{1}}}
$$


Wir schätzen nun auch die Größenordnung der Konstanten $a$ und $b$ ab. Nach (5.3) und (5.4) genügen diese folgenden Beziehungen:

$$
\begin{gathered}
\int_{0}^{\infty} \frac{f(v)(v-b)}{a^{2}+(\nu-b)^{2}} d v=v_{0}-b-2 \pi C_{1} a, \\
a\left[1+\int_{0}^{\infty} \frac{f(v)}{a^{2}+(\nu-b)^{2}} d v\right]=2 \pi C_{1} b=2 \pi f(b) .
\end{gathered}
$$

Wir untersuchen zunächst die zweite Relation. Der größte Beitrag zum Integral kommt aus der Umgebung von $v=b$, so daß wir für die Integration $f(v)$ durch $f(b)$ ersetzen können.

$$
\int_{0}^{\infty} \frac{f(v)}{a^{2}+(\nu-b)^{2}} d v=\left.f(b) \cdot \frac{1}{a} \cdot \operatorname{arctg} \frac{v-b}{a}\right|_{0} ^{\infty}=\frac{f(b)}{a}\left[\frac{\pi}{2}+\operatorname{arctg} \frac{b}{a}\right] .
$$

Eingesetzt erhalten wir:

$$
a=f(b)\left[2 \pi-\frac{\pi}{2}-\operatorname{arctg} \frac{b}{a}\right] .
$$

Die Zahl $a$ liegt daher zwischen $\frac{3 \pi}{2} \cdot f(b)$ und $\pi f(b)$, ist also immer von der Größenordnung $f(b)=C_{1} b$. Da $C_{1}$ sehr klein ist, dürfen wir $\operatorname{arctg} \frac{b}{a} \operatorname{durch} \frac{\pi}{2}$ ersetzen, so daß

$$
a \approx \pi \cdot f(b) .
$$

Zur Abschätzung von $b$ untersuchen wir das Integral

$$
\int_{0}^{\infty} \frac{f(v)(v-b)}{(\nu-b)^{2}+a^{2}} d \nu=C_{1} \int_{0}^{N} \frac{v(\nu-b)}{(\nu-b)^{2}+a^{2}} d \nu+C \int_{N}^{\infty} \frac{\nu^{-\alpha}(v-b)}{(\nu-b)^{2}+a^{2}} d v .
$$

Wir setzen wiederum $\alpha=2$ und erhalten wie bei der Berechnung von $\gamma(v)$ wegen $a \ll b \ll N$

$$
\int_{N}^{\infty} \frac{\nu^{-2}(\nu-b)}{(\nu-b)^{2}+a^{2}} d \nu \approx \int_{N}^{\infty} \frac{1}{v^{2}(\nu-b)} d \nu \approx 0 .
$$

Andererseits ist

$$
\begin{aligned}
\int_{0}^{N} \frac{\nu(\nu-b)}{(\nu-b)^{2}+a^{2}} d \nu & =\nu-a \cdot \operatorname{arctg} \frac{\nu-b}{a}+\frac{b}{2} \cdot \ln \left[a^{2}+(\nu-b)^{2}\right]_{0}^{N} \\
= & N-a \cdot \operatorname{arctg} \frac{N-b}{a}-a \cdot \operatorname{arctg} \frac{b}{a}+\frac{b}{2} \cdot \ln \frac{a^{2}+(N-b)^{2}}{a^{2}+b^{2}} .
\end{aligned}
$$


Da $a$ gegenüber $b$ und auch gegenüber $N$ klein ist, erhalten wir

$$
\begin{aligned}
\int_{0}^{\infty} \frac{f(v)(v-b)}{a^{2}+(\nu-b)^{2}} d \nu & \approx C_{1}\left[N+b \cdot \ln \frac{N-b}{b}\right]=v_{0}-b-2 \pi C_{1} a \\
v_{0}-b & \approx C_{1}\left[N+b \cdot \ln \frac{N-b}{b}\right] .
\end{aligned}
$$

Nun werden wir noch eine gröbere Näherung durchführen, indem wir alle Glieder, die den Faktor $C_{1}$ enthalten, weglassen. Die Beziehungen $(6.7),(6.8),(6.10)-(6.12)$ gehen dann über in

$$
\begin{gathered}
\operatorname{Re} \gamma(v) \approx \operatorname{Im} \gamma(v) \approx 0 \\
b \approx v_{1} \approx v_{0} \\
a \approx \Delta v \approx \pi \cdot f\left(v_{0}\right) .
\end{gathered}
$$

Die natürliche Linienbreite $2 \cdot \Delta v$ ist somit gleich $2 a=\frac{1}{T} ; \mathrm{d}$. h. sie ist umgekehrt proportional zur mittleren Lebensdauer des angeregten Zustandes.

Abschließend werden wir auch eine Näherung für die Zahl $K\left(p_{1}\right)$, die in der Formel (6.1) vorkommt, herleiten. Nach der Beziehung (5.7) ist nämlich

$$
K\left(p_{1}\right)=\left[1+i \Gamma^{\prime}\left(p_{1}\right)\right] \cdot\left[1-i \Gamma^{\prime}\left(p_{1}\right)^{*}\right]=\left|G^{\prime}\left(p_{1}\right)\right|^{2} .
$$

Nun ist aber nach (5.8)

$$
\begin{aligned}
& \operatorname{Re} G^{\prime}\left(p_{1}\right)=-2 a \int_{0}^{\infty} \frac{f(v)(b-v)}{\left[(b-v)^{2}+a^{2}\right]^{2}} d \nu-2 \pi C_{1} \\
& \operatorname{Im} G^{\prime}\left(p_{1}\right)=1+\int_{0}^{\infty} \frac{f(v)\left[(b-v)^{2}-a^{2}\right]}{\left[(b-v)^{2}+a^{2}\right]^{2}} d \nu .
\end{aligned}
$$

Zur Berechnung des Realteils zerlegen wir die Integration in zwei Schritte und benutzen die Beziehung (2.2) mit $\alpha=2$.

$$
\int_{0}^{\infty} \frac{f(v)(b-v)}{\left[(b-v)^{2}+a^{2}\right]^{2}} d v=C_{1} \int_{0}^{N} \frac{v(b-v)}{\left[(b-v)^{2}+a^{2}\right]^{2}} d v+C \cdot \int_{N}^{\infty} \frac{v^{-2}(b-v)}{\left[(b-v)^{2}+a^{2}\right]^{2}} d v .
$$

Wegen $a \ll b \ll N$ ist

$$
\begin{aligned}
\int_{0}^{N} \frac{v \cdot(b-v)}{\left[(b-v)^{2}+a^{2}\right]^{2}} d v & =\left[\frac{v}{2\left[(b-v)^{2}+a^{2}\right]}-\frac{1}{2 a} \cdot \operatorname{arctg} \frac{v-b}{a}\right]_{0}^{N} \\
& \approx \frac{N}{2(b-N)^{2}}-\frac{1}{2 a} \pi \approx \frac{1}{2 N}-\frac{\pi}{2 a} .
\end{aligned}
$$


Anderseits ist

$$
\int_{N}^{\infty} \frac{\nu^{-2}(b-v)}{\left[(b-v)^{2}+a^{2}\right]^{2}} d v \approx \int_{N}^{\infty} \frac{d \nu}{v^{2}(b-v)^{3}} \approx-\int_{N}^{\infty} \frac{d v}{\nu^{5}}=-\frac{1}{4 N^{4}} .
$$

Somit finden wir

$$
\begin{aligned}
\operatorname{Re} G^{\prime}\left(p_{1}\right) & \approx-\frac{2 a C_{1}}{2 N}+\frac{2 a C_{1} \pi}{2 a}+\frac{2 a C}{4 N^{4}}-2 \pi C_{1} \\
& =-\pi C_{1}-\frac{a C_{1}}{N}+\frac{2 a C_{1} N^{3}}{4 N^{4}}=-\pi C_{1}-\frac{a C_{1}}{2 N} \\
\operatorname{Re} G^{\prime}\left(p_{1}\right) & \approx-\pi C_{1} .
\end{aligned}
$$

Den Imaginärteil von $G^{\prime}\left(p_{1}\right)$ haben wir bereits in $\S 5$ ausgerechnet. Nun benutzen wir auch noch die Näherungen (6.13).

$$
\begin{aligned}
\operatorname{Im} G^{\prime}\left(p_{1}\right) & =\frac{2 \pi C_{1} b}{a}-\frac{b \cdot f(b)}{a^{2}+b^{2}}-\frac{f(b)}{2 a}-\frac{f(b)}{a} \cdot \operatorname{arctg} \frac{b}{a} \\
& \approx \frac{2 \pi f(b)}{a}-\frac{f(b)}{b}-\frac{\pi f(b)}{a} \\
& \approx \frac{\pi f\left(v_{0}\right)}{\pi f\left(v_{0}\right)}-\frac{C_{1} \cdot b}{b}
\end{aligned}
$$

$\operatorname{Im} G^{\prime}\left(p_{1}\right) \approx 1-C_{1}$.

Da $C_{1}$ eine sehr kleine Zahl ist, erhalten wir

$$
K\left(p_{1}\right) \approx \pi^{2} C_{1}^{2}+\left(1-C_{1}\right)^{2} \approx 1
$$

Wir setzen die hergeleiteten Näherungen in die Formeln (6.1), (6.4) und (6.5) ein und erhalten dadurch:

$$
\begin{aligned}
\left|a_{0}(t)\right|^{2} & \approx e^{-2 a t} ;\left.\frac{d\left|a_{0}(t)\right|^{2}}{d t}\right|_{t=0} \approx-2 a \\
|a(\nu, \infty)|^{2} & \approx \frac{|H(v)|^{2}}{\left(v-v_{0}\right)^{2}+a^{2}} \\
I(v) & \approx I_{0} \cdot \frac{a / \pi}{\left(\nu-v_{0}\right)^{2}+a^{2}} .
\end{aligned}
$$

Wenn wir schließlich noch $a$ durch $\gamma / 2$ ersetzen, erhalten wir die üblichen Formeln für die Wahrscheinlichkeiten und die Intensitätsverteilung ([1], S. 184).

Bei der Herleitung der Formeln (6.14) haben wir die in (5.17) und (5.18) auftretenden Integrale über $c_{\beta}$ weggelassen. Diese sind indessen sehr stark konvergent, da $e^{p t}$ für große $t$ und für $\operatorname{Re}(p) \rightarrow-\infty$ sehr rasch gegen 0 strebt, und sollen nicht weiter untersucht werden. 


\section{Literatur}

[1] Heituer, W.: The quantum theory of radiation, 3rd edition. London: Oxford University Press 1954.

[2] Weisskopf, V., u. E. Wigner: Z. angew. Phys. 63, 54 (1930).

[3] Heitlen, W., u. S. T. MA: Proc. Irish Acad. 52, 109 (1948).

[4] Arnous, E., and W. Heitler: Proc. Roy. Soc. London, A 220, 290 (1953).

[5] - S. Zienau u. K. Bledler: Helv. Phys. Acta 24, 279 (1951); 25, 581 (1952).

[6] KälléN, G.: Natürliche Linienbreite, Handbuch der Physik, Bd. V. p. 274. Berlin-Göttingen-Heidelberg: Springer 1958.

[7] Doetsch, G.: Handbuch der Laplace-Transformation, Bd. I, p. 43. Basel: Birkhäuser 1950.

[8] Osqood, W. F.: Lehrbuch der Funktionentheorie, 5. Aufl., Bd. I. Leipzig: Teubner 1928.

[9] Ostrowski, A.: Vorlesungen über Differential- und Integralrechnung, Bde. II, III. Basel: Birkhäuser 1954.

[10] Dinghas, A.: Vorlesungen über Funktionentheorie. Berlin-Göttingen-Heidelberg: Springer 1961. 